\title{
Banquets et anti-banquets dans l'œuvre shakespearienne
}

\section{Geneviève LHEUREUX}

\section{CpenEdition}

\section{Journals}

Édition électronique

URL : http://journals.openedition.org/transtexts/610

DOI : $10.4000 /$ transtexts.610

ISSN : 2105-2549

Éditeur

Gregory B. Lee

Référence électronique

Geneviève LHEUREUX, «Banquets et anti-banquets dans l'œuvre shakespearienne », Transtext(e)s Transcultures 跨文本跨文化 [En ligne], 10 | 2015, mis en ligne le 22 juin 2016, consulté le 19 avril 2019 URL : http://journals.openedition.org/transtexts/610; DOI : 10.4000/transtexts.610

Ce document a été généré automatiquement le 19 avril 2019

(C) Tous droits réservés 


\title{
Banquets et anti-banquets dans l'œuvre shakespearienne
}

\author{
Geneviève LHEUREUX
}

1 Se nourrir est à l'évidence à la fois un besoin physiologique propre à tout être vivant et chez l'homme, une pratique culturelle, inscrite en un temps et un lieu donnés. Cependant, à l'intérieur d'une même société, ce que l'on mange dépend en grande partie de facteurs économiques. Les plus pauvres, à l'époque élisabéthaine, en sont parfois réduits à manger des herbes et des racines pendant les périodes de disette et comme le rappelle l'historien Ian Mortimer ${ }^{1}$, les registres paroissiaux des villes et des campagnes témoignent que l'on meurt encore régulièrement de faim. Les années 1594, 1595, 1596, par exemple, sont marquées par des récoltes particulièrement mauvaises en raison de précipitations très abondantes qui entraînent des inondations et de températures estivales anormalement basses, de sorte qu'à l'issue de ces trois ans, le prix du blé augmente de $170 \%$ par rapport à son cours normal, l'avoine de $191 \%$ et il faut importer du seigle du Danemark. Le Songe d'une nuit d'été, probablement composé entre 1595 et 1596, fait du reste allusion à ces désordres climatiques et à leurs conséquences sur l'agriculture :

Titania : The ox hath therefore stretched his yoke in vain,

The ploughman lost his sweat, and the green corn

Hath rotted ere his youth attained a beard.

Le Songe d'une nuit d'été. II. i. 93- 97

Le recours aux enclosures, qui tend à se généraliser dans certaines régions et transforme les terres arables en pâturages, aggrave encore la situation des classes les plus démunies et conduit parfois à des révoltes paysannes, comme celles de 1607-1608, que Shakespeare transpose et adapte dans sa tragédie romaine, Coriolan. Comme le résume de manière laconique William Harrison ${ }^{2}$, dans sa Description de l'Angleterre, publiée en 1587, «As for the poor, they generally dine and sup where they may ». Cette restriction étant posée, il semble que les pratiques alimentaires sont relativement homogènes dans l'Angleterre de la deuxième moitié du XVIème siècle. On ne se contente plus de deux repas, comme au Moyen-Age. La journée débute par le petit-déjeuner, suivi du dîner, qui se prend aux alentours de $11 \mathrm{~h}$ ou bien un peu plus tard, vers midi pour les marchands de la Cité de 
Londres, puis du souper, vers $5 \mathrm{~h}$ de l'après-midi, ou $6 \mathrm{~h}$, si le dîner est décalé. Le dîner constitue le repas principal. Les plats sont apportés sur la table et chacun se sert à son tour selon le rang qu'il occupe dans la maisonnée. On dépose ensuite les aliments dans son assiette, en métal plus ou moins précieux, ou parfois sur une simple planchette de bois, voire une tranche de pain. On dispose pour ce faire d'un couteau et d'une cuillère, mais, à la différence des Italiens, on n'utilise pas de fourchette, sauf si l'on est un jeune homme fraîchement rentré d'un périple à travers l'Europe, qui ne s'appelle pas encore "Grand Tour ${ }^{3}$ »- au risque de passer pour un prétentieux. ${ }^{4}$ Les verres remplacent peu à peu les chopes mais demeurent très chers, comme en témoigne la querelle entre Christopher Sly et l'aubergiste, dans la première scène de l'induction de La Mégère apprivoisée. Les menus varient au gré des saisons, mais la part des légumes et des fruits y est relativement restreinte car ils sont censés perturber les humeurs - c'est du moins ce qu'affirme Sir Thomas Elyot ${ }^{5}$ dans son traité sur la santé, The Castel of Health, publié en 1536 et réédité à dix-sept reprises, et ce que paraît confirmer le Juge Shallow, qui invite Falstaff à goûter les reinettes de son verger assorties de graines de carvi : «[We] will eat a last year's pippin of mine own graffing, with a dish of caraways " (Henry IV, deuxième partie . V. iii. 2-3). A.R. Humphreys, dans l'édition Arden, cite en note à propos de ce passage, deux manuels de diététique contemporains qui recommandent l'association de graines de cumin, de fenouil ou d'anis pour limiter «la ventosité » consécutive à la consommation de pommes. Cette défiance s'atténue cependant au cours du XVIème siècle avec l'introduction de fruits et de légumes cultivés dans les pays voisins : melons et courges de France, choux-fleurs d'Italie, carottes des Pays-Bas. La tomate, importée du NouveauMonde, et déjà très appréciée dans la péninsule cisalpine, indispose par son odeur et est uniquement utilisée en décoration, puis jetée ou donnée en pâture aux cochons. La pomme de terre fait partie des aliments d'exception et s'accommode dans des préparations sucrées réservées aux banquets - je reviendrai un peu plus tard sur le sens de ce mot. ${ }^{6}$ On ne mange pas de champignons, sans doute pour éviter les empoisonnements accidentels, comme l'indique encore Mortimer : «Mushrumps [forme archaïque de mushrooms] are well-known to Shakespeare and his contemporaries, but they never regard them as food: they consider them more suitable for elves to sit under.$^{7} \mathrm{~A}$ l'inverse, on attribue à certains fruits des vertus médicinales. Les pruneaux, en particulier, protègent, croit-on, des maladies vénériennes, c'est pourquoi on en trouve souvent sur le rebord des fenêtres des lieux de prostitution. Cette habitude donne lieu à une série de méprises dans Mesure pour Mesure. Le fromage, jusque-là réservé aux paysans, n'apparaît sur les tables de la noblesse qu'à partir de 1590 environ, quoiqu'il suscite toujours quelques réticences: dans la deuxième partie d'Henry VI, sans doute composée aux alentours de cette même date, Smith le Tisserand fait référence à l'haleine fétide du rebelle Jack Cade qui se nourrit de fromage grillé. Outre le pain, c'est donc la viande qui constitue l'essentiel de l'alimentation, à tel point que le mot «meat » est synonyme de nourriture. Le satiriste Thomas Nashe ${ }^{8}$, constate dans son Anatomie de l'absurde (1588) : "We [...] eat more meat at one meal than the Spaniard or Italian do in a month". La chair du mouton est considérée comme la plus saine, mais on consomme les animaux d'élevage et le gibier que nous connaissons encore aujourd'hui, auxquels il faut ajouter le cygne, les oiseaux marins, comme le goéland ou la mouette et tout un éventail d'échassiers : la grue, le héron, le butor, le courlis, le chevalier gambette - la liste n'est pas exhaustive. ${ }^{9} \mathrm{La}$ consommation de viande est cependant interdite certains jours - les mercredis, vendredis et samedis ainsi que pendant toute la durée du Carême. Certes, l'Eglise imposait déjà à ses fidèles des jours maigres, qui incluaient aussi l'Avent et la veille de la fête d'un certain 
nombre de saints, bien avant le commencement de l'ère Tudor, mais Elisabeth Ière perpétue la tradition des "fish days », non plus inspirée par une démarche pénitentielle d'ordre spirituel mais dictée par la nécessité économique de soutenir l'industrie de la pêche britannique..$^{10}$ Les contrevenants à ces restrictions s'exposent à des peines assez lourdes (jusqu'à trois mois de prison), mais la querelle qui oppose Falstaff à l'aubergiste, dans la deuxième partie d'Henry $I V$, suggère cependant que les autorités tolèrent parfois quelques entorses :

Fasftaff : Marry, there is another indictment upon thee, for suffering flesh to be eaten in thy house, contrary to the law, for the which I think thou wilt howl.

Hostess : All vict'lers do so. What's a joint of mutton or two in a whole Lent?

Henry IV, deuxième partie. II. iv. 340-345

On ne boit pas d'eau, suspectée, sans doute à juste titre, de propager les maladies. Le lait est réservé aux enfants et aux femmes, le vin, importé de France, d'Espagne, de Grèce, ou d'Allemagne aux plus riches, en raison de son coût très élevé. On le boit généralement additionné de sucre, voire de cannelle ou de gingembre ${ }^{11}$ La bière est par conséquent la boisson la plus répandue et consommée en quantités qui paraissent probablement étonnantes de nos jours (Mortimer ${ }^{12}$, à nouveau, précise que dans les grandes maisons, la ration quotidienne allouée aux domestiques correspond à un gallon, soit plus de 4 litres et demi). Il en existe cependant plusieurs variétés - celle que l'on nomme " ale », produite uniquement à partir d'orge fermentée et impossible à conserver, et celle que l'on appelle "beer ", à laquelle on a ajouté du houblon, qui se garde plusieurs mois et dont le degré varie, selon qu'il s'agisse de « double beer », très alcoolisée ou de « small beer » davantage diluée et destinée au plus grand nombre. Dans la deuxième scène de l'induction de $L a$ Mégère, Christopher Sly, déguisé en aristocrate pendant son sommeil, refuse ainsi de goûter au vin d'Espagne qui lui est offert et réclame à grands cris « the smallest ale » ( $L a$ Mégère apprivoisée. Ind. II. 76), la bière de piètre qualité qui lui est habituelle. De même, le prince Hal, dans la deuxième partie d'Henry $I V$, déplore son goût pour un breuvage indigne de son rang:

Prince : Doth it not show vilely in me to desire small beer?

Poins : Why, a prince should not be so loosely studied as to remember so weak a composition.

Prince : Belike then my appetite was not princely got, for, by my troth, I do now remember the poor creature small beer.

Henry IV, deuxième partie. II. ii. 5-11

Ces deux exemples justifient, je l'espère, la longueur de cette introduction, car s'il est bien entendu que le théâtre est un art mimétique, encore faut-il connaitre la réalité qu'il représente. La description, nécessairement très simplifiée, lacunaire des pratiques alimentaires à l'époque de Shakespeare, révèle cependant que l'on mange et que l'on boit sur la scène du Globe, c'est pourquoi, dans une première partie j'étudierai brièvement les occurrences du verbe "eat" dans l'ensemble du corpus, afin de déterminer plus précisément la nature des aliments consommés, qui dépend, en partie, des circonstances dans lesquelles ils sont consommés. A de rares exceptions près ${ }^{13}$, ce ne sont pas les repas pris au quotidien qui sont mis en scène, mais plutôt des fêtes données à l'occasion d'une victoire militaire, d'un couronnement, d'un mariage, ou encore de retrouvailles. Pourtant ces réjouissances s'avèrent souvent perverties, ainsi que je le montrerai dans une deuxième partie. J'illustrerai ce point par une analyse de trois scènes tirées de Macbeth et conclurai cette communication par un retour sur les images évoquées à travers les références à la nourriture, dont la plupart ont trait à la transgression, à l'interdit, comme 
si se nourrir, acte naturel entre tous, s'avérait en définitive contre-nature dans l'œuvre shakespearienne.

Falstaff est sans doute par sa taille et son embonpoint le plus rabelaisien des personnages de Shakespeare. A la scène iv de l'acte II d'Henry IV, première partie, le prince, qui joue le rôle de son père dans l'une des pièces-dans-la-pièce, le compare tour à tour à une outre ballonnée, un tonneau rempli de vin, un énorme sac de viscères ou encore à un bœuf farci :

Prince : Thou art violently carried away from grace, there is a devil haunts thee in the likeness of an old fat man, a tun of man is thy companion. Why dost thou converse with that trunk of humours, that bolting-hutch of beastliness, that swollen parcel of dropsies, that huge bombard of sack, that stuffed cloak-bag of guts, that roasted Manningtree ox with the pudding in his belly [...] ? Wherein is he good, but to taste sack and drink it? Wherein neat and cleanly, but to carve a capon and eat it?

Henry IV, première partie. II. iv. 440-451

Pourtant, lorsqu'un peu plus tard dans la même scène, il trouve dans les poches de Falstaff endormi un reçu établi par l'aubergiste, il s'étonne de la quantité disproportionnée d'alcool qu'il a absorbée par rapport à la nourriture qu'il a commandée : «O monstrous! but one halfpennyworth of bread to this intolerable deal of sack?» ( Henry IV, première partie. II. iv. 533-534). Il semble en effet que l'on boive davantage que l'on ne mange dans le théâtre shakespearien. Ainsi, dans La Nuit des rois, Sir Toby Belch, dont le nom évoque pourtant les conséquences d'un excès de bonne chère et la corpulence celle de son prédécesseur Falstaff, est régulièrement accusé par les autres personnages d'abuser de la boisson et non de la nourriture. Selon Malvolio, il transforme la maison de sa nièce en taverne («Do ye make an ale-house of my lady's house [...] ? " La Nuit de rois. II. iii. 83-84), ce qui pour la servante Maria, causera sa perte (« That quaffing and drinking will undo you. " La Nuit des rois. I. iii. 13). Ces deux avatars de Pantagruel ne sont du reste pas les seuls à succomber à l'attrait qu'exercent la bière et le vin, puisque, dans la tragédie Antoine et Cléopâtre, l'auguste Octave lui-même reconnaît, au terme de la fête donnée sur la galère de Pompée, qu'il n'est plus maître de son élocution (" mine own tongue / Splits what it speaks » Antoine et Cléopâtre. II. vii. 117-118) et qu'Antoine doit faire transporter à terre Lépide, le dernier membre du triumvirat, dont le teint rubicond ( Antoine et Cléopâtre. II. vii. 3) révèle l'état d'ébriété ( «The third part [of the world] is drunk » Antoine et Cléopâtre. II. vi. 85). J'ai néanmoins choisi de consacrer cette première partie à l'étude du verbe manger, sous toutes ses formes (infinitive, conjuguée et participiale) et non du verbe boire, et ce pour deux raisons principales. Tout d'abord le titre de notre colloque, "Manger, représenter ", m'a incitée à privilégier l'étude de la nourriture, même si manger et boire s'inscrivent l'un comme l'autre dans les pratiques alimentaires. Mais surtout, parce que la consultation des Concordances, qui permet, pour chaque pièce, d'accéder aux mots sélectionnés dans leur contexte d'énonciation, suggère que le verbe " eat » renvoie beaucoup plus fréquemment que le verbe «drink» à des associations métaphoriques. Pour résumer la situation de manière un peu caricaturale, ce que boivent les personnages est généralement mentionné à des fins de caractérisation : le penchant du prince Hal pour la petite bière est sans doute indigne de sa condition, mais il annonce le fameux discours de la St Crépin, où Harry, devenu roi, s'adresse à ses soldats comme à ses frères d'armes («We few, we happy few, we band of brothers » Henry V. IV. iii. 60) ; Falstaff et Sir Toby incarnent l'intempérance, qui fait fi des limites ${ }^{14}$ et des règles à l'image du bonhomme Carnaval dont ils sont en partie inspirés; quant à Octave, il 
oppose l'Antoine héroïque, contraint de boire l'urine de ses chevaux pendant la traversée des Alpes (Antoine et Cléopâtre. I. iv.), à l'Antoine oisif que les « bacchanales égyptiennes » ( Antoine et Cléopâtre. II. vii. 103) ont transformé en sybarite ${ }^{15}$. Les références à la nourriture remplissent parfois la même fonction (dans le cas de Jack Cade, par exemple), mais elles suggèrent aussi d'autres pistes de réflexion, que j'aimerais aborder à présent brièvement.

Les occurrences du verbe « eat » sont répertoriées sous la forme d'un tableau (v. Annexe), dans lequel, si le temps imparti le permettait, il serait souhaitable de faire figurer d'autres mots-clés, en particulier des synonymes de « eat » - « feed », « dine », « sup », « swallow », «chew», ou «taste», par exemple. La colonne de gauche indique combien de fois le verbe « manger » apparaît dans chacune des pièces, classées par ordre alphabétique, mais il m'a paru nécessaire de recourir à un critère d'ordre sémantique pour dégager deux sous-catégories supplémentaires. La colonne du milieu correspond au nombre de fois où le verbe est utilisé dans son sens premier, c'est-à-dire quand il signifie ingérer des aliments. Lorsque l'objet du verbe est explicité, il figure sous le nombre d'occurrences de " eat", mais le verbe est parfois construit sans complément, ce qui explique, le cas échéant, la différence entre le nombre d'occurrences et les objets cités. A titre d'exemple :

Sir Toby : [Let] us therefore eat and drink.

La Nuit des rois. II. iii. 12

8 La colonne de droite inclut toutes les références qui ne relèvent pas d'un usage strictement littéral (par exemple, «I will not eat my word» Comme il vous plaira. V. iv.). Elles représentent environ $40 \%$ du total. Il est cependant parfois difficile de trancher entre les deux catégories. C'est ainsi qu'Orlando, dans Comme il vous plaira, reproche à son frère de le forcer à manger la nourriture d'ordinaire réservée aux cochons («husks »), tandis que dans la première partie d'Henry $I V$, Falstaff avoue avoir recruté une armée de pauvres hères faméliques réduits eux aussi à se contenter de la pâtée dont on nourrit les porcs («draff and husks »). Dans les deux cas, il s'agit bien pour les personnages du sens littéral du verbe manger, mais le contexte général évoque simultanément, sur un mode ironique, la parabole du fils prodigue : dans Comme il vous plaira, les rôles sont intervertis, le fils aîné, chargé par son père de prendre soin de son cadet, lui désobéit et soumet Orlando aux privations qui sont le lot du fils rebelle dans le récit biblique; dans Henry IV, les soldats ne sont pas appelés à sacrifier le veau gras, mais comme le prédit Falstaff, à devenir eux-mêmes victimes sacrificielles, chair à canon, « food for powder » (Henry IV, première partie. IV. ii. 65). L'examen des résultats présentés dans le tableau fait en outre apparaître un autre problème: la fréquence d'emploi de «eat» dans chaque œuvre ne correspond pas systématiquement à l'importance accordée à la question de la nourriture sur un plan thématique. Ainsi, Coriolan, dont l'intrigue met en scène des émeutes causées par la famine, comme je l'ai rappelé en introduction, ne compte que cinq références au verbe manger. A l'opposé, la pièce historique Henry $V$, figure au premier rang, avec dixsept références, mais sept d'entre elles consistent en la répétition de « eat a leek » ou bien « eat my leek », proférées par le capitaine Fluellen, déterminé, à la suite d'un différend, à faire avaler tout cru à l'enseigne Pistol, un poireau, emblème national de son Pays de Galles natal. La courte scène (Henry V. V. i.), située au tout début du dernier acte, sert d'intermède comique, mais relève d'une sous-intrigue très périphérique puisque la pièce montre comment le souverain anglais parvient à conquérir le royaume de France. On touche ici encore aux limites du procédé, qui permet malgré tout de formuler quelques remarques. 
Il montre tout d'abord que « eat » est attesté au moins une fois dans la quasi-totalité des pièces : 36 sur 37 , seule la troisième partie d'Henry VI fait exception. Sans doute n'y a-t-il rien d'étonnant à ce constat, puisque manger est une pratique naturelle autant que culturelle, et par conséquent commune à tous les personnages. Il parait en revanche plus surprenant qu'ils recourent en guise de complément à des locutions vagues, approximatives, dépourvues dans la plupart des cas de qualificatifs : «the food», «our / your victuals ", « our meal », « my dinner», "supper», «meat» (qui, on l'a vu, ne désigne pas uniquement de la viande), tout comme "viands » et "cheer ", ou même «flesh» ou «fish» qui font référence à une classe plutôt qu’à un aliment particulier. Certes, les textes s'avèrent parfois plus spécifiques : parmi les fruits et les légumes, on trouve le raisin, les mûres, les pommes reinettes, les pruneaux, le fenouil, associé au congre (dans Henry IV, deuxième partie, c'est là l'unique exemple d'un semblant de recette), de l'ail et des oignons; parmi les viandes, un chapon, du mouton, et du porc. Il faut ajouter à cette liste, un œuf, des crevettes, et du miel. La récolte semble néanmoins bien maigre, au regard de l'étendue du corpus. Où est par exemple le gibier que Shakespeare, braconnier averti dans sa jeunesse si l'on en croit la tradition, avait peut-être lui-même traqué dans la forêt d'Arden? La poursuite de la lecture du tableau suggère une explication. L'homme fait en effet parfois figure de proie plutôt que de prédateur: Antigonus, dans Le Conte d'hiver est attaqué, puis dévoré par un ours; les amants ${ }^{16}$, dans Comme il vous plaira, le roi ${ }^{17}$, ou encore Polonius dans Hamlet sont, une fois mis en terre, digérés par les vers. L'être humain est même à l'occasion la proie de sa propre espèce, mangé par ses semblables, à l'image des chevaux de Duncan, ou de la truie qui a avalé sa progéniture et dont le sang est versé dans le chaudron par les sorcières de Macbeth. Le tableau fait ainsi apparaître trois cas de cannibalisme: dans Périclès, les pratiques anthropophages demeurent hypothétiques - les mères, précise Cléon, gouverneur de Tarse où sévit la famine, sont prêtes à dévorer leurs enfants ("Those mothers [...] are ready now / To eat those little darlings whom they love » Periclès. iv. 42-44) mais la flotte du héros, chargée de blé, vient secourir à temps les affamés ; dans Othello (I. iii. 143-144), elles sont dans une certaine mesure mises à distance, d'abord parce qu'elles s'inscrivent dans un récit, certes donné pour vrai, ensuite parce que le protagoniste dépeint les mœurs des habitants de contrées lointaines («all my travels' history » Othello. I. iii. 139). En revanche, dans Titus Adronicus, la reine Tamora mange ses propres fils, après que Titus a tué les jeunes princes et préparé avec leur chair un pâté pour venger le viol et la mutilation de sa fille Lavinia. Ce dernier exemple, tiré d'une tragédie composée au tout début ${ }^{18}$ de la carrière de Shakespeare, pourrait être décrit comme un anti-banquet, expression que j'aimerais définir rapidement avant d'aborder la deuxième partie de cette présentation.

10 En anglais contemporain, comme en français, le banquet désigne un repas d'apparat, où sont en principe réunis un nombre assez considérable de convives. A l'époque élisabéthaine, le terme ne fait pas référence au repas de fête lui-même mais à une collation, généralement servie après le repas principal, qu'Onions ${ }^{19}$ définit ainsi : "dessert, course or slight repast of fruit and sweetmeats ». J'emploierai néanmoins le mot dans son acception actuelle ${ }^{20}$, qui traduit les termes de " feast » ou " solemn supper " dans l'œuvre de Shakespeare. Traditionnellement, le banquet est destiné à célébrer, et par là même renforcer, les liens qui unissent les participants. Or, dans les pièces, ces événements sont fréquemment détournés de leur fonction première, leur déroulement est interrompu par des assassinats (on ne compte pas moins de quatre meurtres successifs 
en l'espace de vingt lignes dans Titus Andronicus), ou bien sert à l'élaboration de complots. Le procédé n'est certes pas nouveau et est sans doute inspiré de la Cène, puisque c'est au cours de son dernier repas que Jésus annonce la trahison de Judas : «En vérité je vous le dis, l'un de vous me livrera, un qui mange avec moi [...] c'est l'un des Douze qui plonge avec moi la main dans le même plat » (Marc, 14, 18-20). Le motif est ainsi repris, une fois encore sur un mode ironique, dans la scène d'Antoine et Cléopâtre citée précédemment. Il s'agit littéralement du dernier repas de Pompée («the Last Supper» est l'équivalent anglais de la Cène) puisqu'il disparaîtra ensuite de l'intrigue et que l'on apprendra à l'acte III qu'il a été assassiné par l'un des officiers d'Antoine. Cependant, il n'est pas victime d'une trahison, mais plutôt invité à trahir ses alliés romains, à larguer les amarres et une fois en mer, tuer chacun des membres du triumvirat. Menas, l'auteur de ce plan, propose par trois fois à Pompée de devenir le maitre du monde («Wilt thou be lord of all the world ?» Ant. II. vii. 57-65), en d'autres termes, de dominer la Création, à l'égal des dieux. L'épisode est fondé à l'évidence sur le récit de la tentation du Christ au désert qui vient se superposer à celui de la Cène, si ce n'est que Satan apparaît à Jésus au terme de quarante jours de jeûne et que Menas s'adresse à Pompée au beau milieu d'un banquet. Pompée renonce finalement au projet de Menas, mais la scène illustre bien la manière dont la portée symbolique du banquet est dévoyée. C'est en ce sens que la fête devient un antibanquet, comme le masque, ce spectacle aristocratique, qui combine théâtre, poésie, musique et danse, se transforme, sous la plume de Ben Jonson, en anti-masque, son contrepoint grotesque.

11 Macbeth, dont l'intrigue est ponctuée par trois banquets, ou plutôt trois anti-banquets, démontre à quel point ces images scéniques sont efficaces pour suggérer la perversion d'un ordre perçu comme naturel. Le premier (I. vii.) est donné en l'honneur du roi Duncan et se déroule dans les murs de Dunsinane, le château de Macbeth. Les didascalies liminaires suggèrent que le repas bat son plein : « Hautboys and torches. Enter, and pass over the stage, a Sewer, and divers Servants with dishes and service. Then enter Macbeth ». Pourtant, le banquet n'est pas montré, il est remplacé par un long monologue de Macbeth, travaillé par le désir de tuer Duncan, afin de s'emparer de la couronne. Le personnage est seul en scène, à l'écart des festivités, isolé, comme le sont souvent les «villains » du théâtre élisabéthain. Il est pourtant sur le point d'abandonner ses desseins quand il est rejoint par Lady Macbeth, qui le rappelle à son serment :

Lady Macbeth : [...] I have given suck, and know

How tender 'tis to love the babe that milks me:

I would, while it was smiling in my face,

Have pluck'd my nipple from his boneless gums,

And dash'd the brains out, had I so sworn

As you have done to this.

Macbeth. I. vii. 54-59

12 La mère nourricière devient mère meurtrière, "fair is foul and foul is fair " pour reprendre l'incantation des sorcières qui traverse la tragédie. La catégorie des genres se brouille, Macbeth est trop rempli du lait de la tendresse humaine (« Yet do I fear thy nature /It is too full o'th'milk of human kindness » I. v. 16-17), tandis que Lady Macbeth, qui a perdu les attributs de son sexe, ne mettra plus au monde que des enfants mâles :

Macbeth : Bring forth men-children only!

For thy undaunted mettle should compose

Nothing but males.

Macbeth. I. vii. 73-75 

secret par Macbeth, et se tient cette fois-ci sur la scène. En apparence, l'ordre règne à nouveau en Ecosse, chacun occupe la place due à son rang ( "You know your own degrees, sit down » III. iv. 1), le roi boit à la santé de ses hôtes et s'apprête à s'asseoir, au milieu de la table (« Both sides are even : here I'll sit i' th' midst » III. iv. 10), mais il en est empêché par le spectre de Banquo («The table's full» III. iv. 44), qui fait de lui un proscrit. Macbeth, incapable de dormir depuis qu'il a tué Duncan dans son sommeil, est incapable de manger, parce qu'il a fait assassiner Banquo qui se rendait à son invitation. A la fin de la scène, l'harmonie est rompue, le désordre s'est à nouveau emparé du royaume :

Lady Macbeth : [...] At once, good night : -

Stand not upon the order of your going,

But go at once.

Macbeth. III. iv. 117-119

Le chaos atteint cependant son comble dans la scène dite du chaudron (IV. i.), au cours de laquelle les sorcières se préparent à célébrer leur sabbat. Le renversement symbolique, dans ce troisième anti-banquet, parait complet. Les trois sœurs, comme elles se désignent elles-mêmes, se sont retirées sur la lande, loin de toute communauté humaine, pour concocter un infâme brouet, dont les ingrédients sont tenus pour être autant de poisons : crapaud, serpent, œil de têtard, doigt de grenouille, poils de chauve-souris, langue de vipère, crochet d'orvet, patte de lézard, aile de chouette, écaille de dragon, dent de loup, mâchoire de requin, sang de babouin ou encore racine de cigüe et bouture d'if. Elles y ajoutent des morceaux prélevés sur des corps humains, comme si elles destinaient leur breuvage à un rite anthropophage :

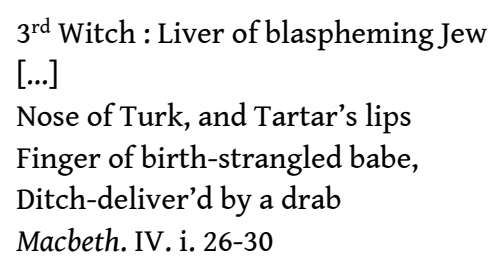

L'omission des articles devant les termes de "Jew», "Turk», «Tartar», et «babe» suggère que chacun d'entre eux ne représente pas un individu mais une catégorie, dont les membres ont en commun de n'être pas baptisés et se trouvent de ce fait exclus du salut. Le bouillon élaboré dans le chaudron, qui mêle plantes et animaux potentiellement mortels aux restes d'humains voués à l'enfer, constitue une sorte d'anti-Création, dans laquelle le vivant, découpé, fragmenté, a perdu toute intégrité. L'image scénique évoque certes le sort de Macbeth, dont les mains menacent de lui arracher les yeux (II. ii. 58) ou celui de Lady Macbeth, condamnée chaque nuit à rejouer dans son sommeil le meurtre de Duncan (V. i.), comme si son corps était dissocié de son esprit, mais elle suggère que ce processus de désagrégation, qui constitue l'un des principaux ressorts tragiques de la pièce, est susceptible de s'étendre à tout le royaume.

17 Les trois anti-banquets qui ponctuent Macbeth s'écartent donc de manière de plus en plus saisissante, au fur et à mesure que progresse l'intrigue, du banquet tel qu'il est traditionnellement représenté. De la mixture empoisonnée que préparent les sorcières surgiront des apparitions qui convaincront Macbeth de se comporter en tyran («Be bloody, bold, and resolute» IV. i. 79), ce qui lui vaudra le rejet de son peuple et 
précipitera sa chute. Macbeth, le «boucher » de l'Ecosse ( V. i. 35), reconnait qu'il a « soupé de l'horreur » (V.v. 13) avant de se lancer dans la bataille qui lui sera fatale.

Les métaphores sont éloquentes. L'acte de se nourrir, indispensable au maintien de la vie, est associé à la mort, le banquet invite même parfois à consommer la chair d'individus de sa propre espèce. L'imaginaire shakespearien détourne ainsi un acte naturel par excellence en un acte contre-nature, qui constitue avec l'inceste, auquel il est du reste lié, une forme ultime de transgression. ${ }^{21}$ En d'autres termes, Shakespeare semble avoir spontanément recours à des images anthropophages lorsqu'il donne à voir un ordre naturel perverti, ou perçu comme tel. C'est ainsi que Richard II, pour plaire à son peuple, renonce à incarner l'autorité souveraine et se laisse dévorer des yeux par ses sujets, qui se font prédateurs et se repaissent du spectacle monarchique jusqu'à l'écœurement :

King : The skipping King, he ambled up and down

[...]

Enfeoff's himself to popularity,

That, being daily swallow'd by men's eyes,

They surfeited with honey, and began

To loathe the taste of sweetness [...]

Being with his presence glutted, gorg'd, and full.

Henry IV, première partie. III. ii. 60-84

Contraint d'abdiquer par son cousin Henry Bolingbroke, il déclenche un cycle de violence qui évoque à nouveau un banquet monstrueux puisque l'Angleterre se gorge du sang de ses enfants (« No more the thirsty entrance of this soil / Shall daub her lips with her own children's blood» Henry IV, première partie. I. i. 5-6). La mère nourricière n'est plus seulement mère infanticide, pour reprendre l'image évoquée par Lady Macbeth (I. vii. 54-59), elle dévore sa progéniture en un acte de transgression ultime, dont l'horreur est inégalée.

\begin{tabular}{|l|l|l|l|}
\hline Titre de la pièce & $\begin{array}{l}\text { Occurrences du verbe } \\
\text { EAT }\end{array}$ & Sens littéral & $\begin{array}{l}\text { Sens } \\
\text { figuré }\end{array}$ \\
\hline All's Well that Ends Well & 8 & $\begin{array}{l}6 \\
\text { Objets explicités: } \\
\text { "grape" } \\
\text { "homely meat" }\end{array}$ & 2 \\
\hline Antony and Cleopatra & 6 & $\begin{array}{l}2 \\
\text { "strange flesh" } \\
\text { "meat" }\end{array}$ & 4 \\
\hline As You Like It & 10 & $\begin{array}{l}\text { "men" (eaten by worms) } \\
\text { "husks" }\end{array}$ & 1 \\
“"a grape" & "the food" & \\
\hline
\end{tabular}




\begin{tabular}{|c|c|c|c|}
\hline Cymbeline & 4 & $\begin{array}{l}4 \\
\text { "our victuals" } \\
\text { "cheer" }\end{array}$ & \\
\hline The Comedy of Errors & 2 & 1 & 1 \\
\hline Coriolanus & 5 & 2 & 3 \\
\hline Hamlet & 8 & $\begin{array}{l}5 \\
\text { "a king" } \\
\text { (eaten by a worm) } \\
\text { "the fish" } \\
\text { "Polonius" } \\
\text { "a crocodile" }\end{array}$ & 3 \\
\hline Julius Caesar & 2 & $\begin{array}{l}2 \\
\text { "your dinner" }\end{array}$ & \\
\hline $\begin{array}{l}\text { King Henry IV, } \\
\text { Part One }\end{array}$ & 5 & $\begin{array}{l}3 \\
\text { "blackberries" } \\
\text { " a capon" } \\
\text { "draff and husks" }\end{array}$ & 2 \\
\hline $\begin{array}{l}\text { King Henry IV, } \\
\text { Part Two }\end{array}$ & 13 & $\begin{array}{l}6 \\
\text { "prawns" } \\
\text { "this letter" } \\
\text { (steeped in sack) } \\
\text { "pippin [...] with a dish of caraways" } \\
\text { "conger and fennel" } \\
\text { "flesh" }\end{array}$ & 7 \\
\hline King Henry V & 17 & $\begin{array}{l}12 \\
\text { "my leek / a leek" (x7) } \\
\text { "your victuals" }\end{array}$ & 5 \\
\hline $\begin{array}{l}\text { King Henry VI, } \\
\text { Part One }\end{array}$ & 2 & $\begin{array}{l}1 \\
\text { "thee"[Joan la Pucelle] } \\
\text { (eaten by a wolf) }\end{array}$ & 1 \\
\hline $\begin{array}{l}\text { King Henry VI, } \\
\text { Part Two }\end{array}$ & 7 & $\begin{array}{l}5 \\
\text { "grass" (x2) } \\
\text { "meat" } \\
\text { "toasted cheese" }\end{array}$ & 2 \\
\hline King Henry VIII & 1 & 1 & \\
\hline King John & 2 & & 2 \\
\hline
\end{tabular}




\begin{tabular}{|c|c|c|c|}
\hline King Lear & 4 & $\begin{array}{l}4 \\
\text { "fish" } \\
\text { "the meat [of an egg]" } \\
\text { "dried oats" } \\
\text { "the swimming frog" } \\
\text { "cow dungs" }\end{array}$ & \\
\hline King Richard II & 4 & 2 & 2 \\
\hline King Richard III & 1 & & 1 \\
\hline Love's Labour's Lost & 2 & & 2 \\
\hline Macbeth & 5 & $\begin{array}{l}4 \\
\text { "[horses eating] each other" } \\
\text { "our meal" } \\
\text { "the insane root" } \\
\text { "[a sow that has eaten] nine of her } \\
\text { farrows" }\end{array}$ & 1 \\
\hline Measure for Measure & 5 & $\begin{array}{l}4 \\
\text { “mutton" } \\
\text { "prunes" }\end{array}$ & 1 \\
\hline The Merchant of Venice & 2 & 2 & \\
\hline $\begin{array}{l}\text { The Merry Wives of } \\
\text { Windsor }\end{array}$ & 4 & 2 & 2 \\
\hline $\begin{array}{l}\text { A Midsummer Night's } \\
\text { Dream }\end{array}$ & 3 & $\begin{array}{l}1 \\
\text { "onions", "garlic" }\end{array}$ & 2 \\
\hline $\begin{array}{l}\text { Much Ado about } \\
\text { Nothing }\end{array}$ & 11 & 3 & 8 \\
\hline Othello & 3 & $\begin{array}{l}1 \\
\text { "[the Cannibals that eat] each other" }\end{array}$ & 2 \\
\hline Pericles & 4 & $\begin{array}{l}3 \\
\text { "[mothers eat] little darlings whom } \\
\text { they love" } \\
\text { "honey" } \\
\text { "viands" }\end{array}$ & 1 \\
\hline Romeo and Juliet & 1 & & 1 \\
\hline
\end{tabular}




\begin{tabular}{|c|c|c|c|}
\hline $\begin{array}{l}\text { The Taming of the } \\
\text { Shrew }\end{array}$ & 8 & 7 & 1 \\
\hline The Tempest & 2 & 2 & \\
\hline Timon of Athens & 13 & $\begin{array}{l}7 \\
\text { "meat" } \\
\text { "root", "roots" }\end{array}$ & 6 \\
\hline Titus Andronicus & 5 & $\begin{array}{l}5 \\
\text { "[Tamora eating] the flesh [of her } \\
\text { children]" }\end{array}$ & \\
\hline Troilus and Cressida & 9 & 2 & 7 \\
\hline Twelfth Night & 3 & 2 & 1 \\
\hline $\begin{array}{l}\text { The Two Gentlemen of } \\
\text { Verona }\end{array}$ & 2 & & 2 \\
\hline The Winter's Tale & 4 & $\begin{array}{l}3 \\
\text { "the gentleman" [eaten by the bear] } \\
\text { "adders' heads and toads } \\
\text { carbonadoed" }\end{array}$ & 1 \\
\hline
\end{tabular}

\section{NOTES}

1. Ian Mortimer, The Time Traveller's Guide to Elizabethan England, 2012, London, Vintage Books, 2013, pp 241-243.

2. Liza Picard, Elizabeth's London, 2003, London, Phoenix, 2004, p.179.

3. L'expression semble attestée à partir de 1670.

4. Mortimer, op.cit. , pp. 246-249.

5. Ibid, p. 245.

6. Ibid, p. 257.

7. Ibid, p. 258.

8. Liza Picard, op.cit. , p179.

9. Mortimer, op. cit. , pp. 251-253.

10. Ibid, p. 244.

11. Ibid, pp. 260-261.

12. Ibid, pp. 262-263. 
13. v. par exemple La Mégère apprivoisée, IV. i. ou Comme il vous plaira, II. vii.

14. Sir Toby : I'll confine myself no finer than I am.

La Nuit des rois. I. iii. 9

Falstaff : I live out of order, out of all compass.

Henry IV, première partie. III. Iii. 18-19

15. Caesar: This is the news: he fishes, drinks, and wastes

The lamps of night in revel; is not more manlike

Than Cleopatra, nor the queen of Ptolemy

More womanly than he [...].

Antoine et Cléopâtre. I. iv. 4-7

16. Rosalind : [Men] have died from time to time and worms have eaten them, but not for love.

Comme il vous plaira. IV. i. 101-130

17. King : Now, Hamlet, where's Polonius?

Hamlet : At supper. [...] Not where he eats, but where a is eaten. A certain convocation of politic worms are e'en at him. [...] A man may fish with the worm that hath eat of a king, and eat of the fish that hath fed of that worm.

Hamlet. IV. iii. 16-28

18. La date de Titus Andronicus est controversée. Un in-quarto fut publié en 1594, mais une remarque de Ben Jonson, qui se moque, en 1614, de ceux qui admirent la pièce depuis vingt-cinq ou trente ans, suggère qu'elle aurait pu être composée bien avant, entre 1585 et 1589.

19. C.T. Onions, A Shakespeare Glossary, Oxford, Oxford University Press, 1986.

20. La situation est en réalité plus complexe. Lorsque le roi Duncan emploie le terme de «banquet » de manière métaphorique (I. iv. 56), il semble faire référence au repas de fête tout entier, ainsi que le remarque Kenneth Muir.

21. Certains bestiaires de la Renaissance indiquent que les serpents se nourrissent de la chair de leurs parents et cette pratique est liée à l'inceste. C'est ce que montre l'énigme que doit déchiffrer Périclès, au début de la pièce :

I am no viper, yet I feed

On mother's flesh which did me breed.

Périclès. I. i. 107-108

Le passage est en effet immédiatement interprété par le héros comme une référence codée à des pratiques incestueuses :

And she, an eater of her mother's flesh

By the defiling of her parents' bed.

Périclès. I. i. 172-173

\section{RÉSUMÉS}

Se nourrir est à l'évidence à la fois un besoin physiologique propre à tout être vivant et, chez l'homme, une pratique culturelle, inscrite en un temps et un lieu donnés, susceptible d'être représentée au théâtre. De fait, le verbe manger (« eat») est attesté dans la quasi-totalité des pièces de Shakespeare, mais lorsqu'il n'est pas employé métaphoriquement, il est rarement suivi d'un complément. Peut-être est-ce parce que l'homme passe parfois du statut de prédateur à celui de proie, dont se repaissent ses semblables. Macbeth met en scène de tels banquets contre- 
nature, ou anti-banquets, qui témoignent de la perversion de l'ordre naturel (ou perçu comme tel) en Ecosse. L'imaginaire shakespearien transforme ainsi l'alimentation, essentielle au maintien de la vie, en un acte mortifère, qui constitue une forme ultime de transgression.

No living creature is exempt from the necessity of feeding themselves, but in the case of mankind, eating is a cultural practice that varies from time to time and place to place and, as such, can be represented on stage. Indeed, the verb "eat", with very few exceptions, occurs in all of Shakespeare's plays, but when it is not used metaphorically, it rarely has an object. The reason might be that sometimes man is eaten rather than eats, and falls prey to his fellow creatures. Macbeth stages such unnatural banquets, or anti-banquets, which reveal that what is seen as the natural order has been perverted. Shakespeare's imagination transforms a life-giving act into a deadly one, which stands for an ultimate form of transgression. 